\title{
Un feu d'artifice ! Un foyer permanent ?
}

\section{J.-C. Debongnie}

(C) Springer-Verlag 2009

Depuis la nouvelle rédaction, c'est-à-dire depuis le congrès de Fès en 2008, plus de 100 articles ont été soumis. Un véritable feu d'artifice! La rédaction est presque noyée. Nous peinons à trouver assez d'experts pour tout analyser. Nous prenons du retard, et nous nous en excusons. Nous comprenons mal que plus de $10 \%$ des auteurs, après s'être donnés la peine d'écrire un article, ne répondent pas aux critiques !

Les cas cliniques servent de rappel. Les articles originaux sont plus importants, car reflétant souvent une série de cas et une discussion du problème en Afrique. C'est le cas dans ce numéro de l'article sur l'intérêt de l'échographie dans le diagnostic de la cirrhose en milieu tropical.

Ce feu d'artifice reflète un enthousiasme et un besoin. L'enthousiasme de pouvoir écrire dans un journal africain adressé à des confrères d'Afrique. Le besoin d'exprimer l'expérience en Afrique, de partager les problèmes, etc. et peut-être de dégager des solutions. Ce numéro l'illustre.
F. Bossali décrit l'état de l'endoscopie dans son pays et ses problèmes. À discuter au prochain congrès de Yaoundé... ou ailleurs ? Deux articles traitent des brûlures caustiques du tube digestif supérieur. Il y a 30 ans, c'était un problème fréquent en Europe de l'Ouest. Il a quasi disparu grâce à de nouvelles précautions d'emballage des caustiques. Ce sujet sera traité à Yaoundé lors d'une session de pédiatrie (S. Cadranel).

Ce feu d'artifice doit être transformé en foyer permanent. Nous comptons sur vous, par exemple sur les gastroentérologues camerounais dont les communications lors du prochain congrès pourraient faire l'objet d'articles. Nous comptons vous aider, par exemple, par un atelier de rédaction à Yaoundé.

L'enthousiasme ne suffit pas..., et l'argent manque : la revue est en déficit. Un nouvel objectif pour notre association : assurer la pérennité, permettre que le foyer reste allumé. 